\title{
PARASITOSES INTESTINAIS: CONTROLE E PREVENÇÃO
}

\author{
Maria do Carmo Barretto de Carvalho*
}

CARVALHO, M. do C.B. de Parasitores intestinais: controle e prevenção. Rev. Esc. Enf. USP, São Paulo, 24(1):117-130, abr. 1990.

A autora se propõe a mostrar a atuação da Enfermeira na Comunidade de Pedregal-Goiás, junto a 50 (cinqüenta) familias que apresentavam uma alta incidência c'e parasitoses intestinais, através de um programa de orientação, em grupo, sobre o controle e prevenção dessa patologia.

UNITE!MMOS: Assistência primária en saide. Parasio'ogio.

\section{INTRODUÇÃO}

A idéia dessa pesquisa surgiu da necessidade sentida durante a realização da disciplina: Metodologia do processo de Enfermagem, do curșo de Enfermagem - UnB, onde o tempo de atuação (cerca de 2 meses) na comunidade de Vila do Paranoá foi insuficiente para a consolidação de uma assistência primária à saúde.

A princípio, o trabalho seria desenvolvido na Vila do Paranoá - Distrito Federal, porém, em virtude da Universidade de Brasília estar desenvolvendo atividades de Extensão e Pesquisa na área de Novo Gama - Goiás, identificou-se a necessidade de aplicar a pesquisa na área mencionacia devido às semelhanças existentes entre esta região e a Vila do Paranoá, no que se refere ao aspecto Saúde.

Distante cerca de $40 \mathrm{Km}$ do Plano Piloto, o Novo Gama compreende três áreas habitacionais distintas: Novo Gama, Pedregal e Céu Azul.

A comunidade de Pedregal com 15.000 habitantes foi escolhida por apresentar maior semelhança com a Vila do Paranoá e por suas características estruturais. No Pedregal, apesar de haver algumas habitações de alvenaria, a grande maioria das residências é construída de madeira. A população é pobre e desprovida de recursos básicos. A água, geralmente, é retirada para o consumo através de cisternas (muitas das quais coletivas) existentes no quintal, não passando por nenhum tratamento.

As parasitoses intestinais constituem um sério problema de saúde, principalmente em comunidades do tipo acima descrito. Segundo DAVIS ${ }^{3}$, a transmissão da maioria das infecções intestinais depende dos niveis locais de saneamento e do fornecimento e qualidade de água. Mediante observação c estudo da situação da comunidade de Pedregal, pôde ser

\footnotetext{
- Enfermeira do Hospital das Doenças do Aparelho Locomotor - SARAH -- Bolsista do CNPq.
} 
constatada a carência ampla de serviços básicos de nutrição e saúde, bem como uma forte suposição de alta infestação de parasitas intestinais na população.

Atualmente a política do Setor de Saúde tem mostrado a necessidade de extensão de cobertura dos serviços de saúde e o emprego da assistência primária e participação comunitária como uma estratégia para alcançar este objetivo.

Segundo os pensamentos de MAHLER 7, cabem ao povo o direito e o dever de participar ativamente da manutenção de sua própria saúde, e em casos de doença, de prover seu próprio tratamento.

Ainda segundo recomendação da ORGANIZAÇÃO MUNDIAL DA SAUUDE ${ }^{9}$, as comunidades devem ser incentivadas a assumir a responsabilidade por sua saúde e bem-estar, necessitando, para tanto, de participar na avaliação da situação, na definição de problemas, na fixação de prioridade, no planejamento de atividades e, finalmente, na execução de tais atividades.

Portanto, os objetivos deste trabalho compreendem: identificar a incidência de parasitoses intestinais, detectar sintomas e sinais de parasitemia, controlar e prevenir as parasitoses intestinais através da educação em saúde.

\section{DESENVOLVIMENTO}

\section{Material e Método}

Com início em julho de 1986, participaram do progranıa 50 (cinqüenta) familias (escolhidas aleatoriamente) residentes no Pedregal. Utilizou-se para a coleta de dados: formulários (vide anexo 1) e exames parasitológicos de fezes.

Para melhor compreensão, dividiu-se o trabalho em 03 (três) fases:

$1^{\text {a }}$ Fase - Identificação das parasitoses intestinais, desenvolvida através das seguintes ações:

a) Visitas domiciliares - primeiro contato entre a enfermeira e a familia visitada com a finalidade de promover um melhor relacionamento entre as duas partes, facilitando o desenvolvimento das ações posteriores.

b) Aplicação de formulário - nesse formulário (vide anexo 1) foi prioritária a investigação de sintomas e sinais, tais como mal-estar, cansaço físico e mental, diarréia e/ou constipação, dores abdominais, vômitos, restos alimentares nas fezes, palidez facial, distensão abdominal, sangue nas fezes, febre e inapetência. Além disso procurou-se levantar as características sócio-econômicas da família, as características ambientais e de saúde. 
c) Exame parasitológico de fezes - o método utilizado foi o de sedimentação de HOFFMAN et alii ${ }^{4}$, com a finalidade de pesquisar cistos, ovos e larvas nas fezes. É largamente empregado na rotina de laboratórios clínicos, não só pela simplicidade como também por ser econômico e sensível. Os frascos coletores contelido solução de formol a $10 \%$ foram entregues com antecedência de 01 (uma) semana para a coleta do material à pessoa responsável da família. Todos os exames foram realizados no Laboratório do Núcleo de Medicina Tropical e Nutrição da Universidade de Brasília.

$2^{\text {a }}$ Fase - Educação para a saúde - Essa fase teve início após a análise e interpretação dos dados coletados anteriormente. As famílias (mães e/ou pais) foram então reunidas em pequenos grupos de 10 (dez). Elaborou-se conjuntamente um plano de assistência para controlar e prevenir as parasitoses, onde as seguintes orientações foram enfocadas:

. higiene corpcral;

. higiene ambiental;

- tratamento caseiro da água;

adoção de medidas sanitárias impedindo a deposição de material fecal no solo;

- tratamento dos doentes - as famílias dirigiram-se ao Centro de Saúde, de posse do resultado dos exames, para adquirir os medicamentos da CEME.

Como material didático utilizou-se cartazes, (slides) e outros materiais para demonstração. A exposição foi realizada semanalmente, em horário pré-fixado, no Centro de Saúde, da forma mais dinâmica e simples possível adequada à população envolvida. Caso houvesse necessidade de maior discussão, por vezes, fazíamos duas sessões quinzenais com o mesmo grupo.

$3^{\text {a }}$ Fase - Manutenção do Controle e Prevenção - Ficou estabelecido para esta fase, manutenção do controle e prevenção, que haveria reuniões mensais entre a enfermeira e novos grupos formados através da divulgação feita pelas famílias iniciadoras do trabalho.

A enfermeira tem apenas o papel de direcionar as reuniões, ficando algumas pessoas encarregadas de transmitir as orientações, recebidas anteriormente, às novas famílias.

O material audio-visual utilizado fica à disposição da comunidade que tem a autonomia de marcar reuniões independentemente da presença da enfermeira, sugerindo então novas propostas de reuniōes que incluem orientações sobre Diarréia, Desidratação, Insuficiência Respiratória Aguda, Saúde da Mulher, entre outros. 


\section{Resultados}

Das 50 (cinquienta) famílias entrevistadas, pode-se identificar 288 (duzentos e oitenta e oito) indivíduos, dando como média por família o total de 5,76 individuos.

Em relação à renda familiar mensal, $4(8 \%)$ famílias possuem renda de menos de 01 (um) salário mínimo, $16(32 \%)$ famílias possuem renda de 01 (um) salário mínimo, $18(36 \%)$ possuem renda de 02 (dois) salários, $6(12 \%)$ famílias possuem renda de 03 (três) salários e 6 (12\%) familias possuem renda mensal de mais de 03 (três) salários mínimos (vide anexo 2, tabela I).

A tabela II (vide anexo 2) mostra as características ambientais das familias, tais como:

$37(74 \%)$ residências são construídas de alvenaria, $6(12 \%)$ são construídas de madeira e $7(14 \%)$ residências são mistas de madeira e alvenaria;

a água para o consumo é retirada de cisternas coletivas por 28

(56\%) famílias, enquanto que $22(44 \%)$ famílias possuem sua própria cisterna;

- em relação ao destino dos dejetos, 31 (62\%) familias possuem sua própria fossa. E 19 (38\%) familias utilizam fossas coletivas;

o lixo nessa região possui três diferentes destinos, sendo que 29 (58\%) famílias costumam queimar, $20(40 \%)$ costumam depositai ao solo e apenas $1(2 \%)$ familia enterra o lixo, que é utilizado como adubo.

O gráfıco I (vide anexo 3) demonstra os sintomas referidos pelos 288 indivíduos, quando da aplicação do formulário, sendo que cada indivíduo referiu no mínimo um sintoma, podendo também um mesmo indivíduo ter referido até cinco sintomas. Destes 288 indivíduos entrevistados, 28 recusaram-se a entregar o material para o exame parasitológico de fezes. Assim, de acordo com a tabela III (vide anexo 4), foram realizados 260 (duzentos e sessenta) exames parasitológicos de fezes, sendo que $184(70,8 \%)$ estavam positivos e $76(29,2 \%)$ negativos (ausentes de vermes).

Constatou-se a incidência de mais um tipo de parasita por indivíduo. Assim, verificou-se dos 184 exames positivos, $124(67,4 \%)$ exames acusaram Entamoeba coli; 48 (26,0\%) exames acusaram Ascaris lumbricoides; $44(23,9 \%)$ exames acusaram Giardia lamblia; ;25 (13,5\%) exames acusaram ancilostomideos; $19(10,3 \%)$ exames acusaram Hymenolepis nana; 9 (4,9\%) exames acusararn Trichiuritrichiura; 9 (4,9\%) exames acusaram Endolimax nana e $1(0,5 \%)$ exame acusou Strongyloides stercoralls (vide anexo 4 , tabela IV). 


\section{Discussão}

a) População estudada

A população desde a $1^{\text {a }}$ fase mostrou-se interessada e disposta a cooperar ativamente. Durante as visitas domiciliares sentiam-se à vontade com a enfermeira, que por muitas vezes foi solicitada a opinar sobre assuntos particulares da familia e também a orientar sobre problemas de saúde em geral.

A população também mostrou-se ansiosa pelo resultado dos exames de fezes, os quais não eram feitos a bastante tempo justamente pela dificuldade de marcação desse exame no Hospital mais próximo, e pela demora de sair o diagnóstico.

Do total de individuos contactados (288), apenas 28 recusaramse a coletar as fezes, sendo que 03 (três) referiram já ter feito o exame recentemente. $E$ interessante ressaltar que todos os indivíduos que se recusaram eram do sexo masculino.

Outro fato a ressaltar da população é que essa participou como modelo do audio-vistial utilizado (slides). Assim a população foi ao mesmo tempo modelo e instrumento do audio-visual, o que facilitou a compreensão do mesmo.

b) Trabalho - Tabelas I, II, III e IV; Gráfico I

A tabela I mostra a distribuição da renda familiar mensal, onde $36 \%$ das famílias possuem renda de 02 salários mínimos mensais, o que é confirmado por CARVALHO et alii ${ }^{1}$ e ,MAHLER ${ }^{8}$, quando a baixa renda está associada à incidência de doenças parasitárias.

Na tabela II, destacam-se alguns dados de relevância. Em relação à água, 28 (56\%) famílias utilizam-se de cisternas colctivas, sendo que a água não passa por nenhum tratamento e muitas vezes a cisterna encontra-se em precárias condições de uso. A fossa própria utilizada por $31(62 \%)$ famílias, é construída na sua grande maioria, de madeira e sem os requisitos básicos de estrutura, como a distância, profundidade e localização em relação à cisterna. Embora 58\% das famílias tenham afirmado que queirnam o lixo, essa não foi a realidade encontrada pois muitas vezes para entrarmos no quintal da casa, tínhamos que limpar o lixo existente. Apenas uma família utiliza o lixo adequadadamente como adubo.

Essas características de cuidados e uso da água, fossa e do lixo, descritas anteriormente (vide tabela II), confirmam a positividade dos exames na tabela III, pois dos 260 exames realizados, $184(70,8 \%)$ acusaram algum tipo de parasita.

Segundo COPELMAN ${ }^{2}$, o método de sedimentação pode resultar às vezes em negatividade dos exames, devido a muitos aspectos como qualidade do formol e do material, problemas laboratoriais 
na identificação dos parasitas, entre outros. Porém isto não invalida a utilização desse método, de acordo com KUNZLE et alii ${ }^{5}$.

$\mathrm{Na}$ incidência dos parasitas descritos na tabela IV, pode-se constatar que a alta incidência de $E$. coli, $A$. lumbricoides e $G$. lamblia confirma o mocio de transmissão desses parasitas feito através da ingestão de água e/ou alimentos contaminados por fezes; ingestão de ovos através da terra, alimentos crus e poeira; ingestão de cistos em água contaminada, alimentos ou transferência à boca por mãos contaminadas de fezes, respectivamente, conforme PESSOA et alii ${ }^{10}$.

No gráfico I, encontramos os sintomas referidos pelos indivíduos, onde a diarréia, as dores abdominais, a febre e o cansaço físico e mentul confirmam o diagnóstico anterior da tabela IV..

A pesquisa dos parasitas no exame parasitológico de fezes é considerada importante, sendo que o tratamento deve estar associado a um programa de controle da parasitose, pois as freqüentes reinfestaçōes e os medicamentos debilitam ainda mais o organismo afetado, segundo LUCAS ${ }^{6}$ e MARSDEN ${ }^{8}$.

SLLVA PINTO et alii 11 ressalta que a educação popular, incluindo a educação para a saúde, deve partir da realidade local e o profissional envolvido deve trabalhar com grupos, o que confirma o sucesso e o envolvimento da comunidade na $2^{\mathrm{a}}$ fase do trabalho.

\section{CONCLUSÃO}

A enfermeira tem um papel de destaque em qualquer setor, ressaltando principalmente seu importante papel em saúde pública. Sua função aliađa à participação comunitária, que deve ser sempre estimada, contribuì para as mudanças estruturais vigentes.

A prevenção, portanto, como teoria e prática, deve constar prioritariamente de todo e qualquer planejamento que envolva a saúde de uma comunidade.

CARVAlHO, M. do C.B. de Parasitic disease: control and prevention. Rev. Esc. Enf. USP, São Paulo, 24(1):117-130, Apr. 1990.

The author intends to show the nurse's actuation in a community of Pedregal Goiás involving a group of 50 families with a high incidence of parasitic disease through an orientation program about the control and prevention.

UNITERMS: Primary health care. Parasitology.

\section{REFERENCIAS BIBLIOGRÁFICAS}

1. CARVAlHo, W.S. et alil. Incldencia de parasitose intestinal na popliaçio de Osasco atendida no Serviço de Patologia Clínica da FUSAM. Rev. Brrs. Patul. Clin., Rio de Janeiro, 16(4):164-8, 1980. 
2. COPELMaN, H. Mótodos de exames parasitológicos de fezes: senect.a Rof. Méd., Belo Horizonte, 6(4):43-6, 1983.

3. DAVIS, A. Este mundo dos vermes. Saúde no mundo, Genebra, —2-3, mar. 1984.

4. HOFFMAN, W.A. et alli. Sedimentation concentration method in schistosomiagis mansoni. J. Publ. Health Trop. Med., Puerto Rico, 9:283-98, 1934.

5. KUNZLE, J.B et alif. Parasitose intestinal: avaliacăo da rrevalencia : mótodô diagnóstícos numa amostra da populaçăo de Ribeiråo Preto. kov. Bras. Iratol. Clin., Rlo de Janelro, 16(2):68-8, 1980.

6. LUCAS, A.O. Recent advances in tropical diseases resiarch. Z. Gesamte Inn Med., Ieipzig, $38(10): 49-53,1983$.

7. MAHLER, H. Saúde park todos no ano 2000: a contagem já comecou. Saúde no mundo, Genebra, —:3-4, fev./mar. 1983.

8. MARSDEN, P.D. The treatment and control of parasitis disease. Rov. Infect. Dis., Chicago, 4(4):883-90, 1982.

9. ORGANIZACXo MUNDLAL DA SAUDE. Cuidados primarios de saúnlo: reiatório da Confortncla Internacional sobro cuidados primários de saúde. Alma-Ata, URSS, 6-12 de setembro de 1978. Brasilia, UNICFF, 1979 . $64 \mathrm{p}$.

10. PESSOA, S B. et all. Paradtologia medica. 11. ed. Rlo de Janeiro, Guanabara Koogan, 1982. $873 \mathrm{p}$.

11. SILVA PINTO, S.M.P. da. et alii. Metodologia de assisténcia: uma nova estratégia de educacăo em saúde. Rov. Bras. The., Brasilia, s6(2):177-82, 1983.

\section{BIBLIOGRAFIA CONSULTADA}

BOTER, D. Possibilidades de control de las geohel mintiasis mediante tratamientos en masa. Bal. Chil. Parasitol., Santiago, $84(1 / 2): 39-43,1979$.

CARVALHFiro, J.R. et all. Contribuictio das doencas infecciosas e parasitárias na morbimortalldado de Ribelrăo Preto. Rov. Saúde Públ., Săo Paulo, 13(3):203-7, 1979.

NEVES, A. Programs for the eradication and control for cndemies under the auspices of the amazonian colonization superintendency brief analysis of its activites in 1978 . Rev. Bras. Malariol. Doencas Trop., Rio de Janeiro, 30:123-8, 1978.

RODRIGUEs, K.H. et alli. A enfermagem no projeto de cooperacăo educacional Vila Paranok. Hev. Bras. Ent., Brasilia, $\$ 6(2): 183-92,1983$.

SAUDE DA COMUNIdAdE: um desaflo. Så Paulo, Paulinas, 1984. 32sp.

SCOTNEY, N. Fducaclio para a caúde: manual para o peseoal de safide da sona rural. Săo Paulo, Paulinas, 1884. 156p.

THAI, D.D. Goals and perspectives of scientific research in medical perasitolgy in developing countrles. Bull. Aaad. Natl. Med., Paris, 168(1/2):262-6, 1984. 
ANEXO 1

QUESTIONARIO

\section{I - CARACTERISTIOAS DA COMUNIDADE}

Nome:

Estado:

Populacia:

Area:

\section{II - CARACTERTSTICAS DA FAMILIA}

Rua:

No:

\section{COMPONENTES}

\begin{tabular}{|l|l|l|l|}
\hline Nome & Idide & Sexo & Grau de Parentesco \\
\hline & & & \\
\hline & & & \\
\hline & & & \\
\hline & & & \\
\hline & & & \\
\hline & & & \\
\hline & & & \\
\hline
\end{tabular}

III - CARACTERISTICAS SOCIO-ECONOMICAS

RENDA FAMILIAR:

( ) - Menor que 01 Salário Mínimo

( ) - 1 Salário Mínimo

( ) - 2 Sálários Minimas

( ) - 3 Salérios Mírimos

( ) - Mais de 3 Scclários Minimos 


\section{IV - CARACTERISTICAS AMBIENTAIS}

a) Descrição co anib:ente em rclação aos aspectos higiênicos (higieno corporal e ambiental, moscas e beratar, etc.).

b) Habitação:

- Distribuiçz̃o:
( ) cm quadra
( ) por cor.junto
( ) Cesorčcnada
( ) outras

- Tipos:

( ) alvencria ( ) madeira ( ) outros

- Resiciencia - Tipos
( ) particular
(1) coletiva
( ) própria
( ) aígada

c) Abastecimento de água
( ) rode pública
( ) rode privativa
( ) tratada
( ) não tratada
( ) crm fluor
( ) sem flúor
( ) bica
( ) mina
( ) rio
( ) fonte
( ) caminhåo pipa
. ( ) outros qual?

d) Dojetos:

Rede de esgotos: ( ) $\operatorname{sim}$ ( ) não

Tem tratamento de esgoto? ( ) sim ( ) não

Tom fosså̃ ( ) sim ( ) não

Observacão quanto ao local da fossa:

Observação quanto ao local da fossa:

Outros: 
e) Lixo:

( ) coleta pública ( ) coleta privada ( ) outro

Coleta: ( ) diária ( ) semanal ( ) quinzenal

( ) outro

Destino do Lixo:

f) Liuminação:

- Redo Elétrica

$$
\begin{aligned}
& \text { ( ) usina central } \\
& \text { ( ) gerador proprio } \\
& \text { ( ) outros }
\end{aligned}
$$

- Há iluminação púbblica nas ruas?

( ) $\operatorname{sim}($ ) não

\section{V - CARACTERISTICAS DE SAUDE}

a) Padrões alimentares

- idosos:

- criangas

- adolescentes:

- adultos:

b) Como são preparados os alímentos (principalmente vegetais e frutas) ? ( ) cru ( ) cozido

c) Hábitos Infantis:

- Brinca com animais? ( ) $\operatorname{sim}($ ) não

Quais?

- Come terra?

( ) $\operatorname{sim}($ ) não

- Toma banho ciariamente? ( ) sim ( ) não

d) Houve caso de doença em algum membro da familia nos últimos 15 dias?

( ) $\operatorname{sim}($ ) não 


\begin{tabular}{|c|c|c|c|c|}
\hline Nome & Idade & Sexxo & Recorreu ao Médico & Sintomas \\
\hline & & & & \\
\hline & & & & \\
\hline & & & & \\
\hline & & & & \\
\hline & & & & \\
\hline & & & & \\
\hline & & & & \\
\hline & & & & \\
\hline & & & & \\
\hline
\end{tabular}

e) Algum membbro da familia já teve um ou mais desses sintomas?

\begin{tabular}{l|l|l|l}
\hline Sintomas & No de Membros & Idade & Sexo \\
\hline
\end{tabular}

Mal-Estar

Cansaço Fisico e Mental

Diarréia

Constipaça

Dores Abdominals

Vômitos

Restos Alimentares nas fezes

Palidez Facial

Distensão Abcominal

Sangue nas fezes

Febre

Inapetencia

f) Quando ocorre doenga na familia, procura-se médico?

( ) $\operatorname{sim}($ ) năo

g) Emom caso de nâo procurar, recorre-se a quem?

( ) curandeira ( ) vivinha

( ) farmácia

( ) parente

( ) outros 


\section{A NEXO 2}

\section{TABELA I}

Distribuição da renda familiar mensal em 50 (cinquerta) familias.

\begin{tabular}{lcccccc}
\hline $\begin{array}{l}\text { Salário } \\
\text { Minimo (*) }\end{array}$ & <que 1 & 1 & 2 & 3 & $>$ que 3 & Total \\
\hline $\begin{array}{l}\text { No de } \\
\text { Familias }\end{array}$ & 4 & 16 & 18 & 6 & 6 & 50 \\
\hline$\%$ & 8 & 32 & 36 & 12 & 12 & 100 \\
\hline
\end{tabular}

Fonte: Formulário aplicado.

(*) Obs.: Salário Mínimo de Cr\$ 890,00

TABELA II

Características ambientais das famí ias entrevistadas, segundo tipo de residencia, uso de água, fossa e destino do lixo.

\begin{tabular}{|c|c|c|c|c|c|c|c|c|c|c|c|c|c|c|}
\hline \multirow{2}{*}{$\begin{array}{c}\text { Caracte- } \\
\text { ristica }\end{array}$} & \multicolumn{3}{|c|}{ Residência } & \multirow{2}{*}{ 朂 } & \multicolumn{2}{|c|}{ Agua } & \multirow{2}{*}{ 是 } & \multicolumn{2}{|c|}{ Fossa } & \multirow{2}{*}{ 㝵 } & \multicolumn{3}{|c|}{ Lixo } & \multirow{2}{*}{ - } \\
\hline & $\mathbf{M}$ & $\mathbf{A}$ & MI & & $\mathbf{P}$ & C & & $\mathbf{P}$ & C & & $\mathbf{Q}$ & $\mathbf{E}$ & D & \\
\hline $\begin{array}{l}\text { No de } \\
\text { Familias }\end{array}$ & 6 & 37 & 7 & 50 & 22 & 28 & 50 & 31 & 19 & 50 & 29 & 1 & 20 & 50 \\
\hline$\%$ & 17 & 74 & 14 & 100 & 44 & 56 & 100 & 62 & 38 & 100 & 58 & 2 & 40 & 100 \\
\hline
\end{tabular}

Fonte: Formulário aplicado.

Legenda: $\mathbf{M}$ - madeira

C - coletiva
A - alveraria
$\mathbf{Q}$ - qucimado
MI - mista
E -.. enterrado
P - própria
D - depositado 


\section{A NEXO 3}

Gráfico I =: Sintomas referidos pelos indivíduos entrevistados

H* DE IPDividuos

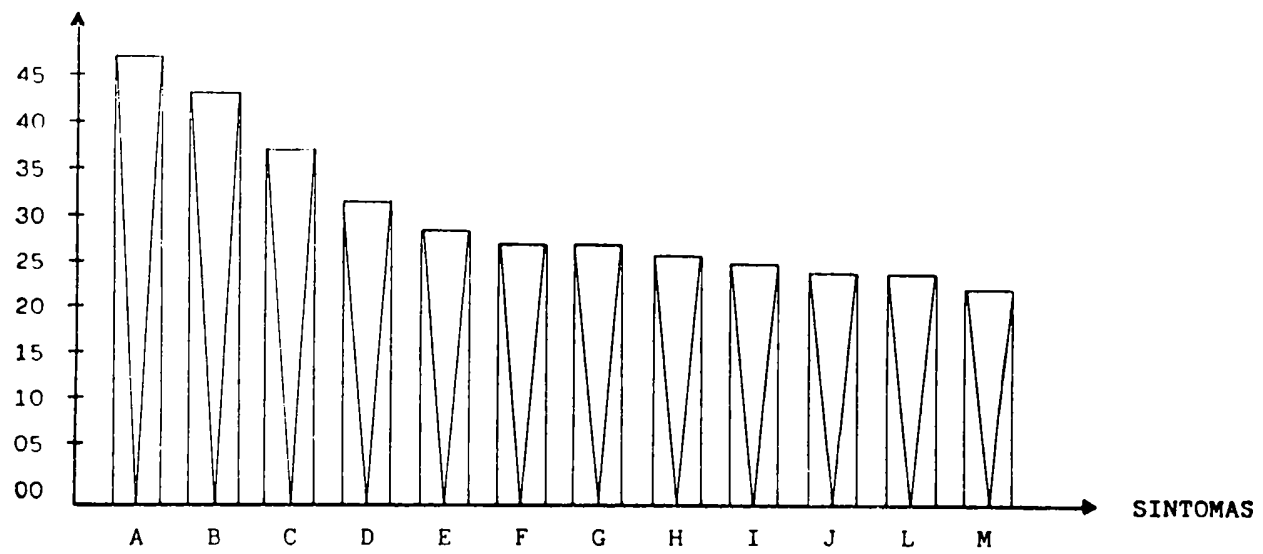

LEG EN D A
A - Diarréia
B - Dores Abdominais
C - Febre
D - Cansaço Físico e Mental
E - Inapetência
F - Constipação

G - Vômitos

H - Mal-estar

I - Distensão Abdominal

$J$ - Sangue nas fezes

L - Restos Alimentares nas fezes

$\mathbf{M}$ - Palidez facial

\section{A NEXO 4}

TABELA III

Positividade dos 260 exames realizados.

\begin{tabular}{lcc}
\hline Exames & Número & $\%$ \\
\hline Positivo & 184 & 70,8 \\
\hline Negativo & 76 & 29,2 \\
\hline Total & 260 & 100,0 \\
\hline
\end{tabular}

Fonte: Exames parasitológicas de fezes 


\section{TABELA IV}

Incidência e distribuiçäo de parasitas intestinais detectados em 184 exames

\begin{tabular}{lcc}
\hline Parasitas detectados & Incidência em 184 exames \\
\hline Entamoeba coli & 124 exames & $67,4 \%$ \\
\hline Ascaris lumbbricoides & 48 exames & $26,0 \%$ \\
\hline Giardia lamblia & 44 exames & $23,9 \%$ \\
\hline Ancilastomideos & 25 exames & $13,5 \%$ \\
\hline Hymenolopis nana & 19 exames & $10,3 \%$ \\
\hline Trichiuris trichiura & 9 exames & $4,9 \%$ \\
\hline Endolimax nana & 9 exames & $4,9 \%$ \\
\hline Strongyloices stercoralis & 1 exame & $0,5 \%$ \\
\hline
\end{tabular}

Fonte: Exames parasitológicas de fezes

Recebido em 29/05/90 